\title{
ESTUDO SOBRE O COMPORTAMENTO MECÂNICO DO POLICARBONATO APÓS DEGRADAÇÃO TÉRMICA*
}

\author{
Cleyson Santos de Paiva ${ }^{1}$ \\ Carlos Alberto Martins Ferreira ${ }^{2}$ \\ Sérgio Neves Monteiro ${ }^{3}$ \\ Verônica Scarpini Candido ${ }^{4}$ \\ Alisson Clay Rios da Silva ${ }^{5}$
}

\section{Resumo}

O comportamento mecânico do policarbonato (PC), da marca PCLIGHT, foi avaliado antes e após a degradação térmica das amostras do polímero em diferentes temperaturas. As amostras de PC em forma de chapa foram expostas por um período de 5 horas nas temperaturas de $50,100,150$ e $200^{\circ} \mathrm{C}$. Posteriormente, foram realizados os ensaios de termogravimetria, tração, flexão e impacto. Os resultados mostraram que as amostras tratadas a $200^{\circ} \mathrm{C}$ tiveram seu desempenho mecânico afetado indicando que a temperatura atua na fragilização do PC. Dessa forma, pode-se inferir que temperaturas acima de $150^{\circ} \mathrm{C}$ podem influenciar negativamente no comportamento mecânico do policarbonato indicando que esse material não deve ter seu uso associado a altas temperaturas.

Palavras-chave: Degradação Térmica, Policarbonato; Propriedades Mecânicas; Polímero.

\section{STUDY ON THE MECHANICAL BEHAVIOR OF POLYCARBONATE AFTER THERMAL DEGRADATION.}

\section{Abstract}

Study of the mechanical behavior of a PCLIGHT brand polycarbonate(PC). It's sample showed interesting results after a thermal degradation in different temperatures. The samples of PC in sheet form were exposed for a period of 5 hours at temperatures of $50,100,150$ e $200^{\circ} \mathrm{C}$. Afther, thermogravimetry test, flexural, impact and tensile tests were performed. The results showed that the samples treated at $200^{\circ} \mathrm{C}$ had their mechanical performance affected indicating that the temperature acts on the PC embrittlement. Thus, it can be inferred that temperatures above $150^{\circ} \mathrm{C}$ can negatively influence the mechanical behavior of the polycarbonate indicating that this material should not have its use associated with high temperatures.

Keywords: Mechanical Properties; Polycarbonate; Polymer, Thermal Degradation.

1 Engenheiro de Produção, Mestre em Ciência e Tecnologia dos Materiais, Rio de Janeiro, Rio de Janeiro e Brasil.

2 Químico industrial, Doutor em Engenharia Metalúrgica e de Materiais, Professor Adjunto I, Curso de Tecnologia em Construção Naval, Centro universitário Estadual da Zona Oeste, Rio de Janeiro - RJ, Brasil.

3 Engenheiro de Materiais, PhD em Engenharia e Ciência dos Materiais, Professor, Programa de Pós-graduação em Engenharia de Materiais/Departamento de Engenharia mecânica e Materiais, Instituto Militar de Engenharia (IME), Rio de Janeiro, Rio de Janeiro, Brasil.

4 Bióloga, Doutora em Ciência dos Materiais, Professora Adjunta I, Faculdade de Engenharia de Materiais, Universidade Federal do Pará (UFPA), Ananindeua-PA, Brasil.

5 Químico industrial, Doutor em Ciência dos Materiais, Professor Adjunto I, Faculdade de Engenharia de Materiais, Universidade Federal do Pará (UFPA), Ananindeua-PA, Brasil.. 


\section{INTRODUÇÃO}

O homem utiliza os recursos naturais para a sua manutenção desde a antiguidade. Ao longo de vários anos e com a necessidade de materiais que suprissem a falta de recursos, foram desenvolvidos produtos sintéticos como os polímeros que utilizam como matéria-prima base os derivados do petróleo [1].

A produção de materiais poliméricos artificiais começou a ser dominado a partir da segunda metade do século XIX, período em que surgiram polímeros modificados, a partir de macromoléculas naturais. A partir do século $X X$, através de estudos de polimerização, começou-se a sintetização de polímeros a partir de unidades básicas de repetição denominadas de mero [2].

Entre os materiais poliméricos, o policarbonato $(\mathrm{PC})$ é um polímero termoplástico, que apresenta boa transparência, elevada resistência térmica e boa ductilidade e tenacidade. É amplamente utilizado em diversos setores industriais, pois combina transparência, elevada resistência ao choque e propriedades óticas, térmicas e mecânicas desejáveis [3,4].

$\mathrm{O}$ PC pertence à família dos poliésteres, que resultam da condensação de poliácidos com poliálcoois. Devido à presença do grupo carbonila (-O-CO-C-) na cadeia principal, esse polímero recebe o nome de policarbonato [1].

Entre os PC, os aromáticos podem ser preparados a partir de vários bisfenóis, sendo o de Bisfenol-A, ou difenil carbonato, o mais importante devido à combinação de diversas propriedades. O policarbonato de Bisfenol-A contém anéis benzênicos e grupos carbonato na cadeia principal e grupos metila ligados à cadeia, onde o grupo funcional cromóforo é o carbonato [5].

Esse polímero é classificado como um termoplástico transparente que apresenta elevada resistência mecânica, estabilidade dimensional ao calor, boa resistência ao fogo, estabilidade química [3]. Ele é amorfo com elevado índice de refração devido a grande quantidade de anéis aromáticos. Ele apresenta a temperatura de fusão cristalina da ordem de $275^{\circ} \mathrm{C}$ e a de transição vítrea de 150 ${ }^{\circ} \mathrm{C}$, sendo considerada elevada quando comparada a outros polímeros [6]. Essa elevada temperatura de transição vítrea pode ser atribuída à restrição de movimento dos seguimentos da cadeia, como 0 anel aromático e as grandes unidades repetitivas [4].

A exposição do policarbonato a elevadas temperaturas pode ser um fator determinante para a diminuição do desempenho desse polímero quando solicitado em serviço [6,7]. Acima de $400^{\circ} \mathrm{C}$ ocorre o rearranjo do grupo carbonato originando produtos como ácidos fenoxibenzóicos e fenilsalicilatos [8]. Reações subsequentes envolvendo a perda de $\mathrm{CO}$ ou $\mathrm{CO}_{2}$ podem levar à formação de diversos éteres e fenóis ou então à reticulação e formação de compostos como xantonas, fenilfenoxibenzoatos e dibenzofuranos. Todas essas reações podem contribuir para a diminuição da tenacidade à fratura desse polímero [7].

Dessa forma, este trabalho teve por objetivo estudar o comportamento mecânico do policarbonato antes e após a uma exposição térmica nas temperaturas de $50,100,150$ e $200^{\circ} \mathrm{C}$ durante 5 horas.

\section{MATERIAIS E MÉTODOS}

O polímero analisado foi uma amostra comercial de policarbonato de BisfenolA (PCLIGHT) produzido pela empresa Policarbonatos do Brasil S. A sob a forma de, fabricada por extrusão. O é produzido industrialmente. 
A partir de chapa de Bisfenol-A com dimensões de $2050 \mathrm{~mm}$ por $1000 \mathrm{~mm}$ e espessura de $6 \mathrm{~mm}$ sem tratamento térmico, produziram-se corpos de prova para ensaios de flexão, impacto Izod e Tração.

Após a etapa de usinagem, foram separados corpos de prova para não exposição térmica e para exposição térmica. Essa exposição se deu nas temperaturas de $50,100,150$ e $200^{\circ} \mathrm{C}$ em forno mufla com taxa de aquecimento de $3,33^{\circ} \mathrm{C} / \mathrm{min}$ e a temperatura de patamar de 5 horas. Posteriormente, desligou-se 0 forno e o resfriamento ocorreu naturalmente.

A tabela 1 apresenta os grupos estudados de acordo com o tratamento térmico recebido.

Tabela 1. Grupos de avaliação

\begin{tabular}{cc}
\hline Grupo de Avaliação & Tratamento Térmico \\
\hline A & Policarbonato sem Tratamento Térmico \\
\hline B & Policarbonato após Tratamento térmico a $50^{\circ} \mathrm{C}$ por 5 horas \\
\hline C & Policarbonato após Tratamento térmico a $100^{\circ} \mathrm{C}$ por 5 horas \\
\hline D & Policarbonato após Tratamento térmico a $150^{\circ} \mathrm{C}$ por 5 horas \\
\hline E & Policarbonato após Tratamento térmico a $200^{\circ} \mathrm{C}$ por 5 horas \\
\hline
\end{tabular}

A análise termogravimétrica (TGA) foi realizada em uma amostra de policarbonato sem tratamento térmico com o auxílio de um analisador térmico da EGA modelo TA Q500 com atmosfera de nitrogênio. Utilizou-se uma taxa de aquecimento de $10^{\circ} \mathrm{C} / \mathrm{mm}$ com uma faixa de variação de temperatura de $27,13^{\circ} \mathrm{C}$ a $790^{\circ} \mathrm{C}$.

Os ensaios de flexão, impacto e tração foram realizados nas amostras sem tratamento térmico e nas amostras que receberam tratamento térmico.

O ensaio de flexão em três pontos foi realizado na temperatura ambiente, seguindo a norma ASTM D 790-10 - Método A [9], em uma máquina universal de ensaios marca EMIC, modelo DL 500N.

O ensaio de impacto Izod foi realizado, à temperatura ambiente, seguindo a norma ASTM D 256-10 - Método A [10], em uma máquina de ensaio Ceast (Resil Impactor), martelo com energia máxima de 7,5J. Foram utilizados corpos de prova com dimensões de $80 \mathrm{~mm} \times 10 \mathrm{~mm} \times 6 \mathrm{~mm}$ e entalhe em $V$ de $45^{\circ}$ e $2 \mathrm{~mm}$ de profundidade fabricados por usinagem mecânica com 0 entalhe produzido em brochadeira.

Os ensaios de tração foram realizados a temperatura ambiente em uma máquina universal de ensaios da marca EMIC, modelo DL 20.000, segundo a norma ASTM D-638 [11], com a taxa de carregamento de $5 \mathrm{~mm} / \mathrm{min}$.

A caracterização fratográfica foi realizada em corpos de prova ensaiados em tração utilizando um microscópio estéreo, marca Olympus modelo SZ61.

\section{RESULTADOS E DISCUSSÃO}

A figura 1 apresenta o comportamento termogravimétrico do Policarbonato sem tratamento térmico. 


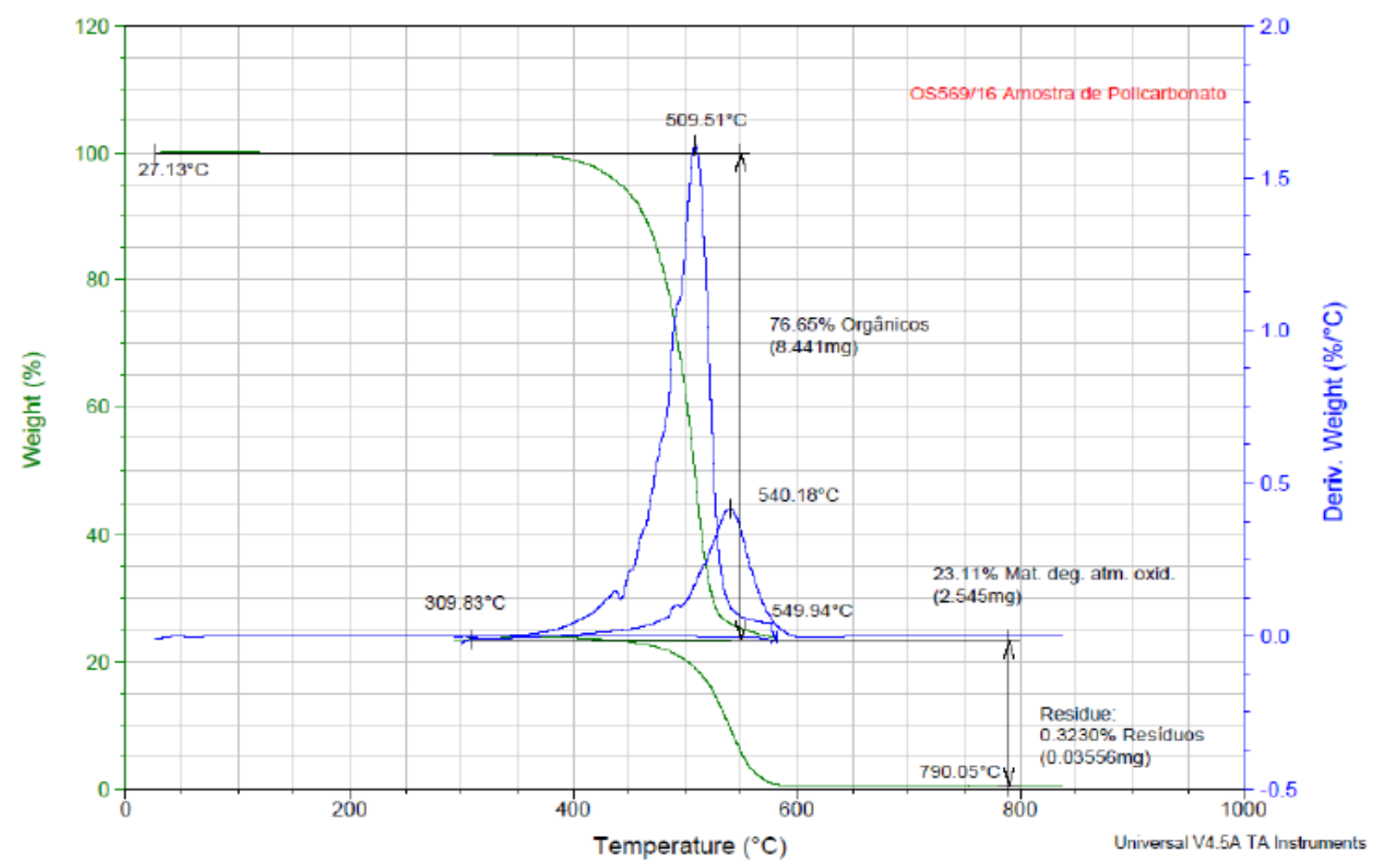

Figura 1. Curva típica de TGA do policarbonato como recebido.

Observa-se que até, aproximadamente, $360^{\circ} \mathrm{C}$ o PC é termicamente estável. Esse comportamento também foi observado por Parvin (1981) [6]. Em 509,51ํㅡ há uma perda de massa de $76,65 \%$ associada à degradação da fração orgânica da cadeia. São observados duas novas perdas de massa a 540,18 e $549,94^{\circ} \mathrm{C}$ associadas, provavelmente, à degradação dos seguimentos inorgânicos da cadeia. Tabela 2.

Os resultados do ensaio de flexão em três pontos são apresentados na

Tabela 2. Propriedades em flexão do policarbonato, antes e após degradação térmica

\begin{tabular}{ccc}
$\begin{array}{c}\text { Grupo de } \\
\text { Avaliação }\end{array}$ & $\begin{array}{c}\text { Tensão de Escoamento } \\
\text { (MPa) }\end{array}$ & $\begin{array}{c}\text { Módulo de Elasticidade } \\
\text { (MPa) }\end{array}$ \\
\hline A & 89 & 2237 \\
B & 89 & 2247 \\
C & 87 & 2145 \\
D & 93 & 2199 \\
E & 73 & 1818 \\
\hline
\end{tabular}

Os valores médios da tensão de escoamento e do módulo de elasticidade das amostras não tratadas termicamente e das amostras tratadas termicamente até $100^{\circ} \mathrm{C}$ são muito similares. O maior valor para essas propriedades foi observado nas amostras tratadas termicamente à $150^{\circ} \mathrm{C}$ e, o menor valor, nas amostras tratadas termicamente à $200^{\circ} \mathrm{C}$ indicando que essa temperatura induz a uma fragilização do material. Esse mesmo comportamento foi por Weber et al. (2006) [5] ao estudarem as propriedades de flexão do policarbonato.

Os resultados de ensaio de impacto Izod das amostras não tratadas termicamente e das amostras tratadas termicamente estão apresentados na Tabela 3. 
Tabela 3. Propriedades em impacto do policarbonato, antes e após degradação térmica

\begin{tabular}{cr}
\hline Grupo de Avaliação & Energia de Impacto \\
A & 626,34 \\
B & 365,37 \\
C & 274,92 \\
D & 215,82 \\
E & 192,26
\end{tabular}

A: Sem tratamento térmico; B: Tratamento térmico à $50^{\circ} \mathrm{C}$; C: Tratamento térmico à $100^{\circ}$; $\mathbf{D}$ :

Tratamento térmico à $150^{\circ} \mathrm{C}$; $\mathrm{E}$ : Tratamento térmico à $200^{\circ} \mathrm{C}$.

Os resultados mostraram que o policarbonato sem tratamento térmico apresenta elevada energia de impacto Izod e os que receberam tratamento térmico tiveram os valores dessa propriedade diminuída. Os menores valores de energia de impacto foram observados nas amostras tratadas a 150 e $200^{\circ} \mathrm{C}$. Esse comportamento indica uma redução da tenacidade provocada, provavelmente, pela cisão das cadeias poliméricas que induz a um rearranjo da cadeia molecular impedindo maior absorção de energia quando uma carga de impacto é aplicada [8, 12]. Essa diferença nos valores de energia de impacto observada no policarbonato evidencia uma transição dúctil-frágil indicando que a temperatura é uma variável que induz a fragilização do material.

Os resultados de tração são apresentados na Tabela 4.

Tabela 4. Propriedades em tração do policarbonato, antes e após degradação térmica

\begin{tabular}{cccc}
\hline $\begin{array}{c}\text { Grupo de } \\
\text { Avaliação }\end{array}$ & $\begin{array}{c}\text { Módulo de } \\
\text { Elasticidade } \\
\text { (MPa) }\end{array}$ & $\begin{array}{c}\text { Tensão no Limite de } \\
\text { Escoamento } \\
\text { (MPa) }\end{array}$ & $\begin{array}{c}\text { Alongamento na } \\
\text { Ruptura } \\
\text { (mm/mm) }\end{array}$ \\
\hline A & 963 & 60 & 0,8 \\
B & 919 & 57 & 0,4 \\
C & 913 & 59 & 0,3 \\
D & 846 & 68 & 0,3 \\
E & 874 & 45 & 0,1 \\
\hline
\end{tabular}

A: Sem tratamento térmico; $\mathbf{B}$ : Tratamento térmico à $50^{\circ} \mathrm{C} ; \mathbf{C}$ : Tratamento térmico à $100^{\circ} ; \mathbf{D}$ :

Tratamento térmico à $150^{\circ} \mathrm{C}$; $\mathrm{E}$ : Tratamento térmico à $200^{\circ} \mathrm{C}$.

Verifica-se que todas as amostras de PC degradadas termicamente tiveram, em relação as amostras sem tratamento térmico, menor alongamento na ruptura indicando uma redução na ductilidade. No que se refere à tensão de escoamento, as amostras tratadas termicamente à $200^{\circ} \mathrm{C}$ obtiveram menor valor para essa propriedade, indicando que a temperatura pode reduzir a resistência à tração do PC. Esses resultados são similares aos encontrados por Turton e White (2001) [13].

As curvas tensão-deformação em tração do $\mathrm{PC}$, antes e após a degradação térmica, estão apresentadas na Figura 2. 


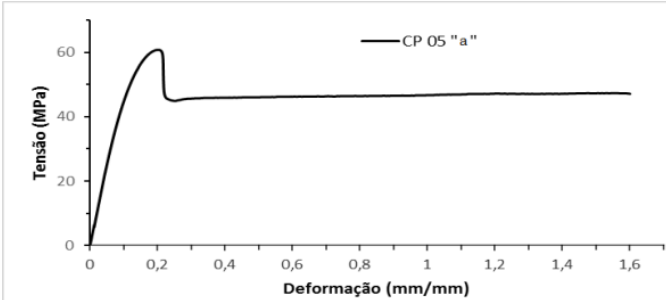

(a)

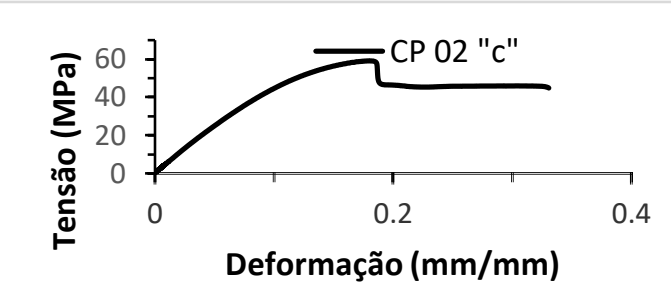

(c)

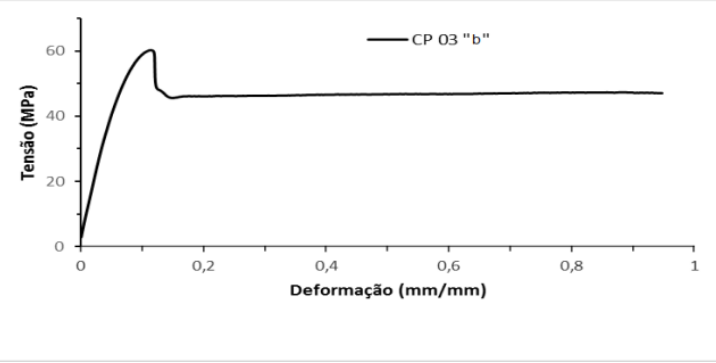

(b)

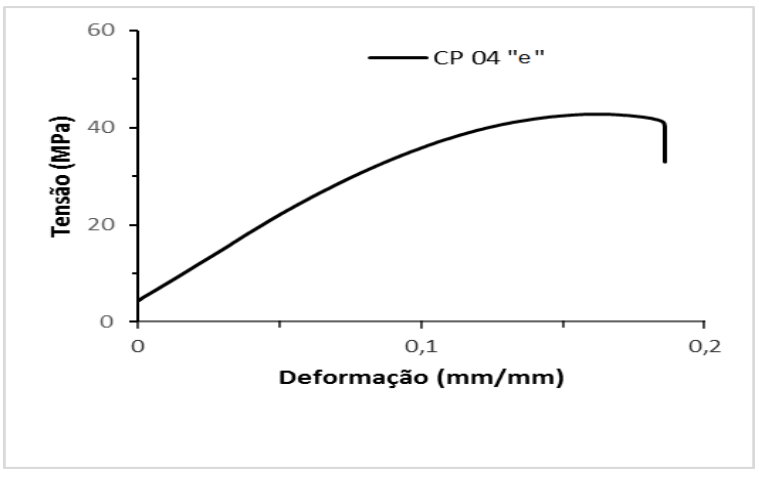

(d)

(e)

Figura 2. Curvas tensão-deformação de engenharia típicas obtidas no ensaio de tração do policarbonato. (a) Sem tratamento térmico (b) Tratamento térmico à $50^{\circ} \mathrm{C}$, (c) Tratamento térmico à $100^{\circ}$, (d) Tratamento térmico à $150^{\circ} \mathrm{C},(\mathrm{e})$ Tratamento térmico à $200^{\circ} \mathrm{C}$.

Observa-se que estas curvas são similares às curvas tensão-deformação características dos materiais poliméricos. Além disso, nota-se um limite superior e inferior de escoamento indicando que esse polímero apresenta um comportamento dúctil.

As micrografias das superfícies de fratura de corpos de prova ensaiados em tração são apresentadas na Figura 4. 


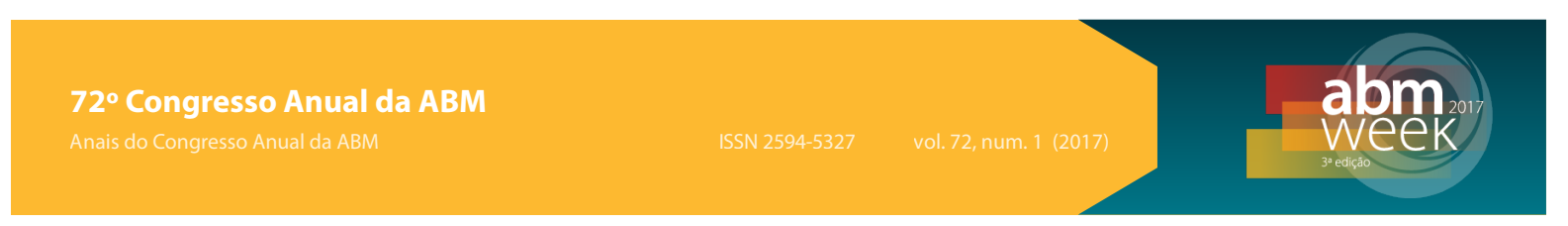

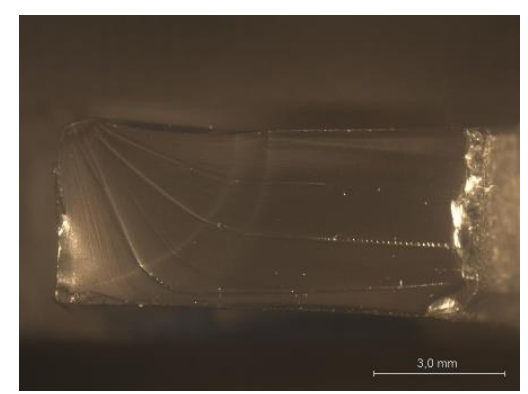

(a)

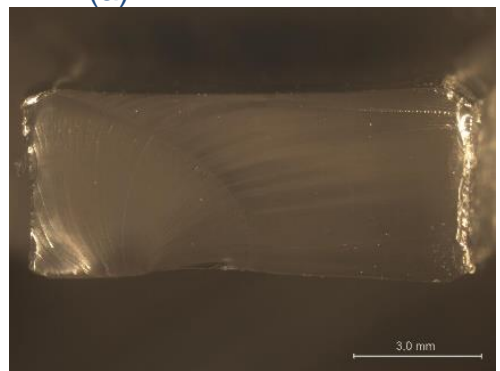

(d)

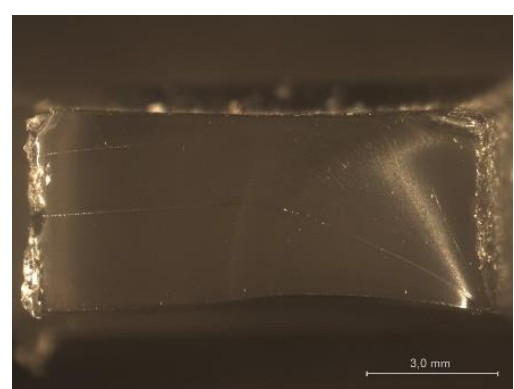

(b)

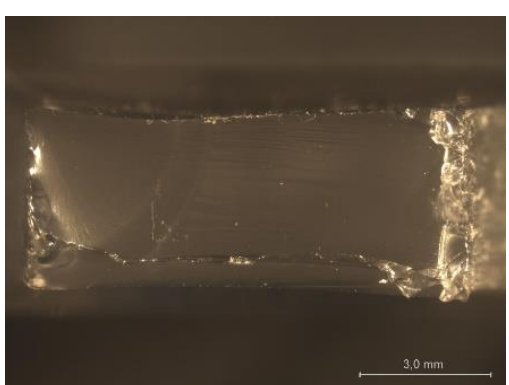

(c)

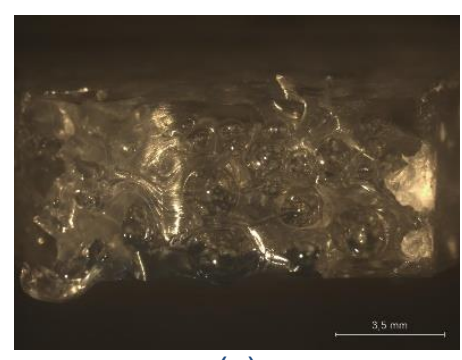

(e)

Figura 4. Aspecto microscópio das fraturas dos corpos de prova com ampliação de 1x: (a) Sem tratamento térmico (b) Tratamento térmico à $50^{\circ} \mathrm{C}$, (c) Tratamento térmico à $100^{\circ}$, (d) Tratamento térmico à $150^{\circ} \mathrm{C},(\mathrm{e})$ Tratamento térmico à $200^{\circ} \mathrm{C}$.

As micrografias revelam que todas as fraturas ocorreram de maneira frágil. Além disso, pode ser observado na fig 4 ((a), (b), (c) e (d)) uma zona espelhada semicircular com tonalidade mais clara que pode ser um indicativo de nucleação e propagação da trinca. Esse padrão caracteriza que a fratura ocorreu no estado plano sendo um aspecto característico de termoplásticos amorfos [13]. Na fig 4 (e) nota-se a presença de microvazios, muito provavelmente, causados pela exposição à temperatura. Esse aspecto reforça os baixos valores das propriedades de tração encontrados para as amostras tratadas a $200^{\circ} \mathrm{C}$ indicando que essa temperatura induz a degradação do policarbonato. Assim, pode-se inferir que a exposição do policarbonato a temperaturas superiores a $150^{\circ} \mathrm{C}$ pode ocasionar na redução das suas propriedades mecânicas, sendo sua utilização não indicada em ambientes com elevadas temperaturas.

\section{CONCLUSÃO}

O estudo sobre o comportamento mecânicos do policarbonato após a degradação térmica revelou:

- O policarbonato é termicamente estável até aproximadamente $360^{\circ} \mathrm{C}$, após essa temperatura são observadas perdas de massa associadas a degradação da cadeia polimérica.

- Amostras tratadas a $200^{\circ} \mathrm{C}$ apresentaram a menor tensão de escoamento quando solicitadas em flexão indicando que essa temperatura pode fragilizar o policarbonato.

- A resistência ao impacto após exposição ao calor reduz a tenacidade do policarbonato tornando-o mais frágil. Esta maior fragilidade pode estar associada à cisão das cadeias poliméricas, reduzindo a massa molecular, 0 que reduz a resistência à propagação da trinca no material.

- Os ensaios de resistência à tração mostraram que a temperatura de $200^{\circ} \mathrm{C}$ prejudica o comportamento mecânico do policarbonato evidenciando que 
esse material não pode ser aplicado em ambientes com temperaturas superiores a $150^{\circ} \mathrm{C}$.

- A análise da região da fratura dos corpos de prova de tração revelou que as fraturas atuantes no policarbonato apresentam aspectos característicos dos mecanismos de fratura, com exceção da amostra exposta a $200^{\circ} \mathrm{C}$ que possui microvazios próximos a superfície onde inicia a trinca, confirmando os resultados numéricos obtidos.

\section{REFERÊNCIAS}

1 Pakull R, Grigo U, Freitag U. Rapra Review Reports-Current Developments in Materials Technology and Engineering, Report 42, Polycarbonates, Pergamon Press, 1991

2 Clough R., Radiation-Resistant Polymers - Encyclopedia of Polymer Science and Engineering. 1988; 13: $667-708$.

3 Turton TJ, White JR. Degradation depth profiles and fracture of UV exposed polycarbonate. Plastics, Rubber and Composite. 2001; 30(4):175-181.

4 Melo NS. Comportamento mecânico do policarbonato exposto à radiação gama. Dissertação de mestrado, Instituto Militar de Engenharia, 2004.

5 Weber RP, Miguez Suarez JC. Behavior of polycarbonate armor: Influence of gamma irradiation. Journal of Physique IV. 2006; 134:941-947.

6 Parvin M. The Effect of Annealing on Fracture Behaviour of Polycarbonate, Journal of Materials Science. 1981; 16: 1796-1800.

7 Saron IC, Felisberti II, MI. Influência de colorantes na degradação termo-oxidativa do policarbonato. Revista Materia. 2009; 14(3):1028-1038.

8 Cho K, Yang J, Kang B, Park CE. Notch sensitivity of polycarbonate and toughened polycarbonate, Journal of Applied Polymer Science. 2003; 89: 3115-3121.

9 American Society for Testing and Materials, ASTM D 790-10. Flexural properties of unreinforced and reinforced plastics and electrical insulating materials, Standard Test Methods for. Philadelphia, PA, 2010.

10 American Society for Testing and Materials. ASTM D256-10 Test Methods for Determining the Izod Pendulum Impact Resistance of Plastics. PA, 2010.

11 American Society for Testing and Materials. ASTM D638-10 .Standard test method for tensile properties of plastics, Philadelphia, PA, 2010.

12 Sekhon GS, Kumar S, Kaur C, Verma NK, Chakarvarti SK. Effect of thermal annealing on pore density, pore size and pore homogeneity of polycarbonate NTFs. Radiation Measurements. 2008; 43: 1357-1359.

13 Passaglia E. Crazes and Fracture in Polymers, J. Phys. Chem. So/ds. 1987; 48(11) 107-1100. 